\title{
Bounding greybody and deflection angle of improved Schwarzschild black hole
}

\author{
Wajiha Javed, ${ }^{1, *}$ Muhammad Aqib, ${ }^{1, \dagger}$ and Ali Övgün ${ }^{2, \ddagger}$ \\ ${ }^{1}$ Division of Science and Technology, University of Education, Township Campus, Lahore-54590, Pakistan \\ ${ }^{2}$ Physics Department, Eastern Mediterranean University, \\ Famagusta, 99628 North Cyprus via Mersin 10, Turkey.
}

(Dated: November 12, 2021)

\begin{abstract}
We explore the deflection angle in the framework of improved Schwarzschild Black hole utilizing the most advance geometrical path adopted by Gibbon-Werner. To investigate deflection angle of the photon ray by weak gravitational lensing for this black hole, we derive the optical curvature and perform the application of Gauss-Bonnet theorem on the optical metric. Moreover, we study the impacts of the plasma medium in context of the weak gravitational lensing in relate to this black hole. Further, we also study the graphical analysis of the deflection angle in both the plasma and non-plasma mediums. Also, we find the rigorous bound base upon the greybody factor for improved Schwarzschild black hole. A while later, we contrast our conclusions about deflection angle with the deflection angles of Schwarzschild black hole within plasma and non-plasma mediums.
\end{abstract}

PACS numbers: 95.30.Sf, 98.62.Sb, 97.60.Lf

Keywords: General Relativity; Improved Schwarzschild Black hole; Plasma medium; Gauss-Bonnet theorem; Non plasma medium; Hawking radiation; Greybody Factor.

\section{INTRODUCTION}

Black holes (BHs) are such things in space that produce deep sinks of gravity, not even light can escape from the powerful pull of a (BH) gravity beyond a certain area. Einstein was the first person who predicted the existence of a $\mathrm{BH}$. The astronomer John Michael was first to originate the idea of $\mathrm{BH}$ [1]. On the basis of hypothesis, astronomers found three types of $\mathrm{BHs}$, named as stellar $\mathrm{BH}$, supermassive $\mathrm{BH}$ and intermediate $\mathrm{BH}$, based on their sizes and masses. The boundary around the BH is known as event horizon, in which photon ray can't escape from it. A particle can't escape if it crosses the event horizon. The spacetime curvature almost infinite at the center of a BH and there occur a gravitational singularity. The phenomenon in which the photon ray released by for away galaxies permits by massive objects present in the entire universe, gravitational attraction from these objects cause the distortion or bending of photon ray, this phenomenon is known as gravitational lensing (GL). GL was initially suggested by solder in 1801, in the background of the theory of Newtonian [2]. Scientist analysis the effect of GL phenomenon that tells about dark matter which is distributed within the distant galaxies. This technique provide us a probe to visualizing both the expansion of the universe and development of the structure in the universe [3]. Photon traverse in the universe and their trajectories are affected by the gravitational effects due to mass concentration. Einstein theory of general relativity (GR) tested with the help of GL, at that point GL worked in different spacetime utilizing alternate strategies [4]-[10]. In 2008, Gibbon and Werner (GW) formulate an arrangement to acquire deflection angle of photon [11]. They consider both the observer and source are present in place of asymptotic Minkowski area. To this procedure, GW used the application of Gauss-Bonnet theorem (GBT) to a spatially space, which is identified with optical metric. In case of GBT, one can use space of $D_{A}$, which one is restricted by photon ray similarly as that of a circular boundary curve $C$ that arranged at the center around the spot of convergence where the beam of the photon ray meets the same source and spectator. It's imagined that the coordinate distance $A$ from the focal point with respect to source and viewer. GBT is carried out in the optical metric, in weak field estimation, as follows;

$$
\iint_{D_{A}} \mathcal{K} d S+\oint_{\partial D_{A}} \kappa d t+\Sigma_{p} \theta_{p}=2 \pi \mathcal{X}\left(D_{A}\right)
$$

Here, $\mathcal{K}$ is the representative of the optical Gaussian curvature and $d S$ is characterized an areal component. Eventually, the value of the Euler characteristic is taken as $\mathcal{X}\left(D_{A}\right)=1$ and further suppose jump angle $\Sigma_{p} \theta_{p}=\pi$, also the

\footnotetext{
*Electronic address: wajiha.javed@ue.edu.pk

${ }^{\dagger}$ Electronic address: msf1900146@ue.edu.pk

‡Electronic address: ali.ovgun@emu.edu.tr
} 
deflection angle obtained by using the following equation acting in uniformity by the straight line estimation:

$$
\eta=-\int_{0}^{\pi} \int_{\frac{b}{r \sin \phi}}^{\infty} \mathcal{K} d S
$$

Therefore, scientists Werner prolonged this methodology for the stationary BHs [12]. Next, Ishihara et al. [13] expressed that the deflection angle is determine for finite distances which means large impact parameter, so the GW barely determine deflection angle with the help of optical fermat geometry technique of the BHs spacetime in the presence of week field limits and also for the spectators having at asymptotically flat section. In recent times, the scientists Crisnejo and the Gallo also reviewed the bending of the photon in the plasma medium [14]. In this way, the weak gravitational lensing gradually taking interest through the technique of GBT for the different BHs and cosmic string and wormholes [15]-[62]. Our main determination of the present article that is to analyze the impact of the deflection angle at improved Schwarzschild BH to which we can apply the GBT and the bending of the light would behave like a global impact. Therefore we merely concentrate at the nonsingular zone outer of the light rays.

Among the most significant aspects of BHs is that they behave like a thermal systems. In particular, BHs sustain entropy and it can release a radiation known as Hawking radiation (HR) [63]-[70]. Consequently, over the event horizon, the spectrum of radiations emitted by BHs is identical to black-body spectrum. Because the spacetime nearby the $(\mathrm{BH})$ is curved type, the spectrum ejected by the $\mathrm{BH}$ has been significantly revised. In this manner, the curvature of spacetime can behave like a potential barrier, allowing some radiation to transmit as well as reflect, as seen in a similar scenario in quantum mechanics. In this sense, the greybody factor is determined to account for transmitting amplitude from the BHs radiations. However, it can also be interpreted as that of the probability for wave going from infinity being absorbed through the $\mathrm{BH}$, which is also known as the probability rate of absorption. In this context, the transmission probability is referred as, the greybody factor. The greybody factor can be calculated in a number of different ways. As for instance, somebody can derive an approximate, greybody factor by utilizing the matching approach. If the value of gravitational potential is sufficiently high, someone utilize the (WKB)approximation approach to obtain the greybody factor [71]-[74]. However apart from approximation, the rigorous bound can be used to calculate the greybody factor [75]-[84]. A black hole's can provide a qualitative depiction.

This new article is summarized it as follows. The Sec. 2, we are talking about optical metric of the spacetime and in Sec. 3, we discuss in detail about GBT and find the value of deflection angle. In the Sec. 4, we discuss about graphically analysis and in Sec. 5, we discuss about impact of plasma on optical metric and find the newly result of deflection angle in presence of plasma after this in Sec. 6, we also draw graphs within plasma and then in the Sec. 7 , we calculate the greybody factor and in the sec.8, we observe its graphical influences and then finally we acquire our consequences.

\section{WEAK GRAVITATIONAL LENSING AND IMPROVED SCHWARZSCHILD BLACK HOLE}

In this scenario, the line element for static and the spherically symmetric optical spacetime is defined as,

$$
d s^{2}=-f(r) d t^{2}+\frac{d r^{2}}{f(r)}+r^{2} d \Omega_{2}^{2}
$$

where the radial coordinate is denoted by $r$, and

$$
d \Omega_{2}^{2}=d \theta^{2}+\sin ^{2} \theta d \phi^{2} .
$$

Here, $d \Omega_{2}^{2}$ denoted the line-element of unit two dimensional sphere and the value of $f(r)$ is defined as, [85, 86]

$$
f(r)=1-\frac{2 M r^{2}}{r^{3}+\tilde{\omega}(r+\psi M)},
$$

where the value of parameters $\psi$ and $\tilde{\omega}$ found in,

after simplifying $f(r)$, we get;

$$
f(r)=1-\frac{\tilde{\omega}^{2}}{r^{4}}-\frac{2 \tilde{\omega}^{2} \psi M}{r^{5}}-\frac{2 M}{r}+\frac{2 M \tilde{\omega}}{r^{3}},
$$


here the mass of $(\mathrm{BH})$ is denoted by $M, r$ is the radial coordinate, $\psi$ is free parameter and $\tilde{\omega}$ is fixed parameter. Putting the value of $f(r)$ in Eq. (1), as a result we obtain the following

$$
\begin{aligned}
d s^{2} & =-\left(1-\frac{\tilde{\omega}^{2}}{r^{4}}-\frac{2 \tilde{\omega}^{2} \psi M}{r^{5}}-\frac{2 M}{r}+\frac{2 M \tilde{\omega}}{r^{3}}\right) d t^{2}+\left(1-\frac{\tilde{\omega}^{2}}{r^{4}}-\frac{2 \tilde{\omega}^{2} \psi M}{r^{5}}-\frac{2 M}{r}\right. \\
& \left.+\frac{2 M \tilde{\omega}}{r^{3}}\right)^{-1} d r^{2}+r^{2} d \theta^{2}+r^{2} \sin ^{2} \theta d \phi^{2} .
\end{aligned}
$$

By applying the basic procedure we let $\left(\theta=\frac{\pi}{2}\right)$ to the moving photon's in the space equatorial plane and also for the null geodesic, we let $d s^{2}=0$, after this we obtain the resulting optical metric which given as follows,

$$
d t^{2}=\frac{d r^{2}}{\left(1-\frac{\tilde{\omega}^{2}}{r^{4}}-\frac{2 \tilde{\omega}^{2} \psi M}{r^{5}}-\frac{2 M}{r}+\frac{2 M \tilde{\omega}}{r^{3}}\right)^{2}}+\frac{r^{2} d \phi^{2}}{\left(1-\frac{\tilde{\omega}^{2}}{r^{4}}-\frac{2 \tilde{\omega}^{2} \psi M}{r^{5}}-\frac{2 M}{r}+\frac{2 M \tilde{\omega}}{r^{3}}\right)} .
$$

Now, we change the optical metric into new coordinate $\hat{r}$, which written as

$$
d t^{2}=g_{a b}^{*} d x^{a} d x^{b}=d \hat{r}^{2}+f^{2}(\hat{r}) d \phi^{2}
$$

where

$$
\begin{aligned}
\hat{r} & =\frac{d r}{\left(1-\frac{\tilde{\omega}^{2}}{r^{4}}-\frac{2 \tilde{\omega}^{2} \psi M}{r^{5}}-\frac{2 M}{r}+\frac{2 M \tilde{\omega}}{r^{3}}\right)}, \\
f(\hat{r}) & =\frac{r}{\sqrt{\left(1-\frac{\tilde{\omega}^{2}}{r^{4}}-\frac{2 \tilde{\omega}^{2} \psi M}{r^{5}}-\frac{2 M}{r}+\frac{2 M \tilde{\omega}}{r^{3}}\right)}} .
\end{aligned}
$$

Now, we changed the expression $(a, b)$ in the $(r, \Phi)$ and the value of the determinant is given as $\operatorname{det} g_{a b}^{*}=\frac{r^{2}}{f(\hat{r})^{3}}$. To using of Eq. (6), only the non-zero christofell values are written as;

$$
\Gamma_{\phi \phi}^{\hat{r}}=\frac{r\left(r f^{\prime}(\hat{r})-2 f(\hat{r})\right.}{2} \quad, \quad \Gamma_{\hat{r} \phi}^{\phi}=\frac{-r f^{\prime}(\hat{r})+2 f(\hat{r})}{2 r f(\hat{r})}=\Gamma_{\phi \hat{r}}^{\phi} \quad, \quad \Gamma_{\hat{r} \hat{r}}^{\hat{r}}=-\frac{f^{\prime}(\hat{r})}{f(\hat{r})}
$$

and Riemann tensor of just only the non-vanishing components for the optical curvature of the space is given as: $R_{\hat{r} \phi \hat{r} \phi}=-k f^{2}(\hat{r})$ where $R_{\hat{r} \phi \hat{r} \phi}=g_{\hat{r} \hat{r}} R_{\phi \hat{r} \phi}^{\hat{r}}$. We can calculate the value of Gaussian optical curvature $(\mathcal{K})$ given it as below;

$$
\mathcal{K}=\frac{R_{\text {icciScalar }}}{2}
$$

Using previous equation, we can rewrite the value of the Gaussian optical curvature $(\mathcal{K})$ which is in the form of the Schwarzschild radial coordinate " $\mathrm{r}$ " written as [? ];

$$
\mathcal{K}=\frac{-1}{f(\hat{r})}\left[\frac{d r}{d \hat{r}} \frac{d}{d r}\left(\frac{d r}{d \hat{r}}\right) \frac{d f}{d r}+\frac{d^{2} f}{d r^{2}}\left(\frac{d r}{d \hat{r}}\right)^{2}\right] .
$$

At the end, we can attain the required value of the Gaussian optical curvature of the photon's from the improved Schwarzschild BH by applying Eq. (7) into Eq. (9), we derive such a formulation

$$
\mathcal{K} \approx-\frac{2 M}{r^{3}}+\frac{12 M \tilde{\omega}}{r^{5}}+\mathcal{O}\left(M^{2}, \tilde{\omega}^{2}, \psi, r^{6}\right)
$$

\section{THE DEFLECTION ANGLE OF IMPROVED SCHWARZSCHILD BLACK HOLE UNDER THE INFLUENCE OF NON-PLASMA}

Now we can calculate the value of deflection angle for the improved Schwarzschild (BH) with the using of GaussBonnet theorem through the existence of non-plasma medium. The GBT is applied in the region of $\mathcal{Y}_{R}$, which is written as [11];

$$
\iint_{\mathcal{Y}_{R}} \mathcal{K} d S+\oint_{\partial \mathcal{Y}_{R}} \dot{k} d t+\sum_{j} \epsilon_{j}=2 \pi \mathcal{X}\left(\mathcal{Y}_{R}\right)
$$


here the term $\mathcal{K}$ denote the Gaussian optical curvature and $k$ denote the value of geodesic curvature, followed in a manner as; $\dot{k}=\dot{g}\left(\nabla_{\dot{\gamma}} \dot{\gamma}, \ddot{\gamma}\right)$ a way that of taking $g(\dot{\gamma}, \dot{\gamma})=1$, where the value of $\ddot{\gamma}$ representative of (the unit acceleration vector), $\epsilon_{j}$ is denote exterior angle to jth vertex. The jump angle become $\pi / 2$ as $\mathrm{R}$ approaches to infinity and yield the term $\left(\theta_{O}+\theta_{S} \rightarrow \pi\right)$ and the value of Euler characteristic $\left(\mathcal{X}\left(\mathcal{Y}_{R}\right)=1\right)$ as $\mathcal{Y}_{R}$ having non singular. Hence, we obtain the following;

$$
\iint_{\mathcal{Y}_{R}} \mathcal{K} d S+\oint_{\partial \mathcal{Y}_{R}} \hat{k} d t+\epsilon_{j}=2 \pi \mathcal{X}\left(\mathcal{Y}_{R}\right)
$$

where the value of the total jump angle is represented by, $\epsilon_{j}=\pi$ and $\gamma_{g}$ is geodesic. Since $\mathcal{X}$ is 1 is denote term of the Euler characteristic number. As, R approaches to infinity the rest of the part yield that $k\left(Z_{R}\right)=\left|\nabla_{\dot{Z}_{R}} \dot{Z}_{R}\right|$. Since, the value of the radial component of geodesic curvature's given it as;

$$
\left(\nabla_{\dot{Z}_{R}} \dot{Z}_{R}\right)^{r}=\dot{Z}_{R}^{\Phi} \partial_{\Phi} \dot{Z}_{R}^{r}+\Gamma_{\Phi \Phi}^{\hat{r}}\left(\dot{Z}_{R}^{\Phi}\right)^{2}
$$

Taking value of $R$ is very large then, $Z_{R}:=r(\Phi)=R=$ const. As a result, the structure of the following equation Eq. (13) changes to $\left(\dot{Z}_{R}^{\Phi}\right)^{2}=\frac{1}{f^{2}\left(r^{\hat{r}}\right)}$. Remembering $\Gamma_{\phi \phi}^{\hat{r}}=\frac{r\left(r f^{\prime}(\hat{r})-2 f(\hat{r})\right.}{2}$, we obtain

$$
\left(\nabla_{\dot{Z}_{R}^{r}} \dot{Z}_{R}^{r}\right)^{r} \rightarrow \frac{1}{R}
$$

As a result, the term geodesic curvature which is not affected by the relevant topological defects. So, $\hat{k}\left(Z_{R}\right) \rightarrow R^{-1}$. Using the relevant optical metric given by Eq. (6), which could be written as $d t=R d$

Phi. So, we conclude that;

$$
\hat{k}\left(Z_{R}\right) d t=d \Phi
$$

Taking all of the above results added together, we achieve [11]

$$
\iint_{\mathcal{Z}_{R}} \mathcal{K} d s+\oint_{\partial \mathcal{Z}_{R}} \dot{k} d t={ }^{R \rightarrow \infty} \iint_{T_{\infty}} \mathcal{K} d S+\int_{0}^{\pi+\grave{\varphi}} d \varphi
$$

At 0th order, the photon beam in the context of weak deflection limits is given it as $r(t)=b / \sin \Phi$. Thus by utilize Eq. (2.13) and the Eq. (17), the formula of deflection angle is written as [11];

$$
\grave{\varphi}=-\int_{0}^{\pi} \int_{b / \sin \varphi}^{\infty} \mathcal{K} \sqrt{\operatorname{detg}^{*}} d \hat{r} d \Phi
$$

where

$$
\sqrt{\operatorname{detg}^{*}}=r+3 M+\frac{3 \tilde{\omega}^{2}}{2 r^{3}}+\frac{3 \tilde{\omega}^{2} \psi M}{r^{4}}-\frac{3 M \tilde{\omega}}{r^{2}} .
$$

At the end, by using the main order values of the relevant Gaussian curvature of Eq. (2.13) in the Eq. (17), the resulting value of the deflection angle is defined as:

$$
\grave{\varphi} \approx \frac{4 M}{b}-\frac{16 M \tilde{\omega}}{3 b^{3}}+\mathcal{O}\left(M^{2}, \tilde{\omega}^{2}, \psi, r^{6}\right)
$$

\section{GRAPHICAL ANALYSIS FOR NON-PLASMA MEDIUM}

This present section devoted to inspect resulted deflection angle by graphically. We also talk about the physically significant of these graphs. Further, we observe the effect of fixed parameter $\tilde{\omega}$ and also about impact parameter $(b)$ on the deflection angle. 
A. Correspondence between the deflection angle $\grave{\varphi}$ and $b$ (impact parameter)

(i)

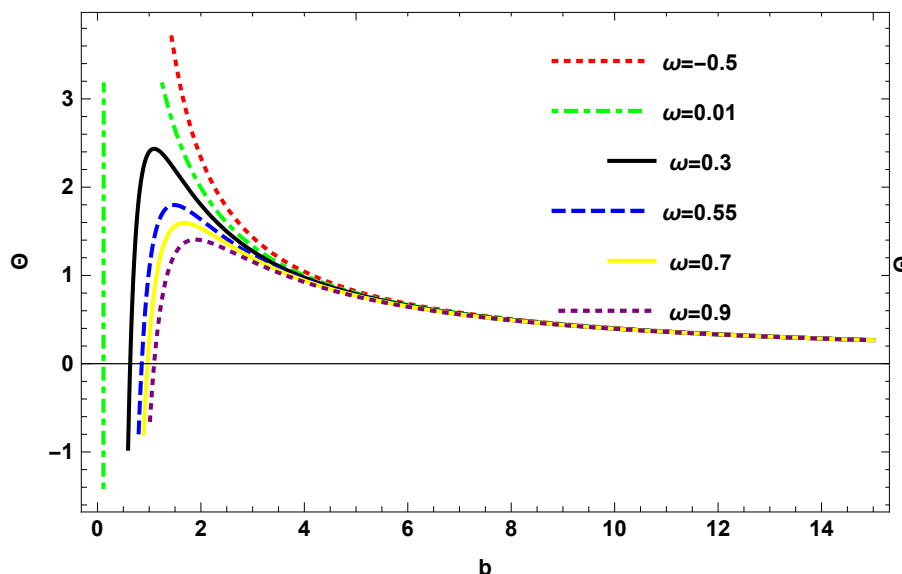

(ii)

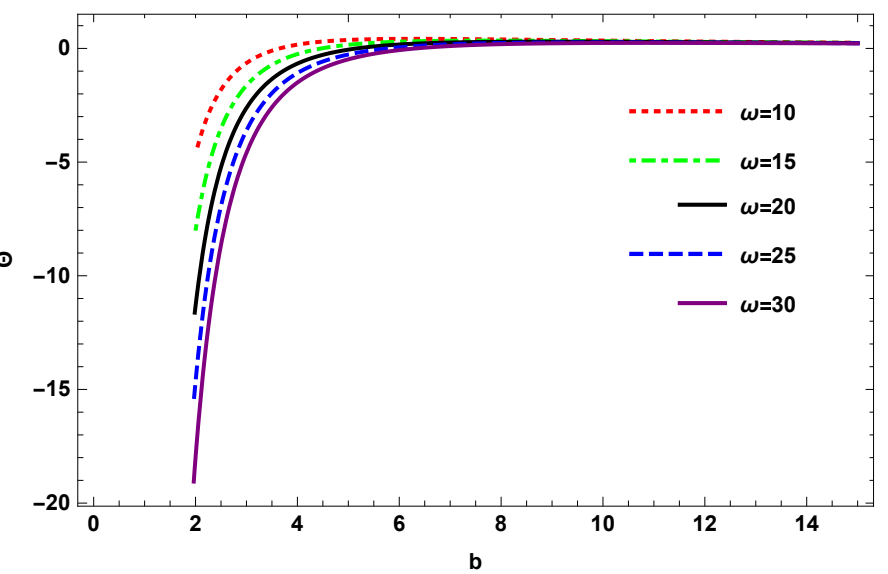

Picture 1: Coordination between $\grave{\varphi}$ and (impact parameter).

- Picture 1 To express the activity of the deflection angle with the value of impact parameter by taking $M$ fixed and alternating the value of parameter $\omega$.

1. In left fig (i), we discovered deflection angle steadily increasing first to keep the lower values of $\omega$.

2. In right fig (ii), we watched the value of deflection angle is successively decline to keep the values of $\omega$ large.

\section{B. Deflection angle $\grave{\varphi}$ with parameter $\omega$}

(i)

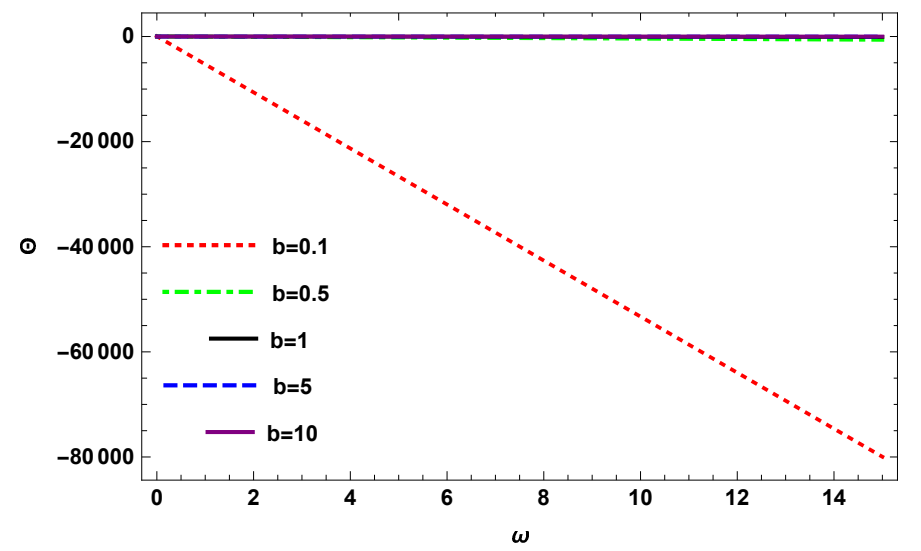

Picture 2: Correspondence between $\grave{\varphi}$ and $\omega$.

- Picture 2: Here manifests the behavior of $\grave{\varphi}$ regarding with $\omega$ to take $M$ fixed and changing value of $b$.

1. In the fig(i), we sees that the deflection angle geometrically decrease first by carrying high values of (impact parameter) $b$ further deflection angle instantly increase when the values between $0.1<b<0.5$.

\section{WEAK LENSING BY IMPROVED SCHWARZSCHILD BLACK HOLE IN CASE OF PLASMA MEDIUM}

To this section, we investigate weak gravitational lensing from improved Schwarzschild (BH) in plasma medium. The value of the refractive index $n$ [14] for improved Schwarzschild (BH) is written by,

$$
n^{2}(r, \omega(r))=1-\frac{\omega_{e}^{2}(r)}{\omega_{\infty}^{2}(r)} .
$$


where $\omega_{e}$ denote the frequency of electron plasma and $\omega_{\infty}$ denote the frequency of photon which is measured by an observer at $\infty$. The value of the (refractive index) to this present situation is illustrated as,

$$
n \dot{(r)}=\sqrt{1-\frac{\omega_{e}^{2}}{\omega_{\infty}^{2}}\left(1-\frac{\tilde{\omega}^{2}}{r^{4}}-\frac{2 \tilde{\omega}^{2} \psi M}{r^{5}}-\frac{2 M}{r}+\frac{2 M \tilde{\omega}}{r^{3}}\right)} .
$$

The value of corresponding metric function is illustrated as,

$$
d s^{2}=-f(r) d t^{2}+\frac{1}{f(r)} d r^{2}+r^{2} d \Omega_{2}^{2}
$$

and

$$
f(r)=1-\frac{\tilde{\omega}^{2}}{r^{4}}-\frac{2 \tilde{\omega}^{2} \psi M}{r^{5}}-\frac{2 M}{r}+\frac{2 M \tilde{\omega}}{r^{3}} .
$$

By assuming that the source and the spectators both of them are on the equatorial plane, null photon direction on an identical surface, so $\left(\dot{\theta}=\frac{\pi}{2}\right)$. In case of null geodesic, we assume that $d s^{2}=0$ and as a result we obtain the corresponding optical metric given as below [14],

$$
d t^{2}=g_{p q}^{o p t} d x^{p} d x^{q}=n^{2}\left[\frac{d r^{2}}{f^{2}(r)}+\frac{r^{2} d \vartheta^{2}}{f(r)}\right]
$$

with determinant $g_{p q}^{o p t}$,

$$
\sqrt{g^{o p t}}=r\left(1-\frac{\omega_{e}^{2}}{\omega_{\infty}^{2}}\right)+\frac{\tilde{\omega}^{2}}{2 r^{3}}\left(3-\frac{\omega_{e}^{2}}{\omega_{\infty}^{2}}\right)+\frac{\tilde{\omega}^{2} M \psi}{r^{4}}\left(3-\frac{\omega_{e}^{2}}{\omega_{\infty}^{2}}\right)+M\left(3-\frac{\omega_{e}^{2}}{\omega_{\infty}^{2}}\right)-\frac{M \tilde{\omega}}{r^{2}}\left(3-\frac{\omega_{e}^{2}}{\omega_{\infty}^{2}}\right) .
$$

Taking into count Eq. (23), the christofell symbols of non-zero values are demonstrated as,

$$
\begin{gathered}
\Gamma_{00}^{0}=\left(1+\frac{\omega_{e}^{2} f(r)}{\omega_{\infty}^{2}}\right)\left[-f(r)^{\prime} f(r)^{-1}\left(1-\frac{\omega_{e}^{2} f(r)}{\omega_{\infty}^{2}}\right)-\frac{f(r)^{\prime} \omega_{e}^{2}}{2 \omega_{\infty}^{2}}\right], \\
\Gamma_{10}^{1}=\left(1+\frac{\omega_{e}^{2} f(r)}{\omega_{\infty}^{2}}\right)\left[r^{-1}\left(1-\frac{\omega_{e}^{2} f(r)}{\omega_{\infty}^{2}}-\frac{f(r)^{\prime} f(r)^{-1}}{2}\left(1-\frac{\omega_{e}^{2} f(r)}{\omega_{\infty}^{2}}\right)-\frac{f(r)^{\prime} \omega_{e}^{2}}{2 \omega_{\infty}^{2}}\right]\right.
\end{gathered}
$$

and

$$
\Gamma_{11}^{0}=\left(1+\frac{f(r) \omega_{e}^{2}}{\omega_{\infty}^{2}}\right)\left[-r f(r)\left(1-\frac{f(r) \omega_{e}^{2}}{\omega_{\infty}^{2}}\right)+\frac{r^{2} f(r)^{\prime}}{2}\left(1-\frac{f(r) \omega_{e}^{2}}{\omega_{\infty}^{2}}\right)+\frac{r^{2} f(r)}{2} \frac{f(r)^{\prime} \omega_{e}^{2}}{\omega_{\infty}^{2}}\right]
$$

Hence, by using the non-zero christofell symbols values given above, then corresponding value of Gaussian optical curvature in the context of curvature tensor is written as,

$$
\mathcal{K}=\frac{R_{r \vartheta r \vartheta}\left(g^{o p t}\right)}{\operatorname{det}\left(g^{\text {opt }}\right)}
$$

To utilize Eq.(24) following Gaussian curvature is determined as,

$$
\mathcal{K} \approx-\frac{2 M}{r^{3}}+\frac{12 M \tilde{\omega}}{r^{5}}-\frac{3 M \omega_{e}^{2}}{r^{3} \omega_{\infty}^{2}}+\frac{21 M \tilde{\omega} \omega_{e}^{2}}{r^{5} \omega_{\infty}^{2}}+\mathcal{O}\left(M^{2}, \tilde{\omega}^{2}, \psi, r^{6}\right)
$$

We derive the corresponding deflection angle with help of the GBT which related to non-plasma. In this way, we can also find the value of deflection angle in weak fields zone, such as photon rays obeys a straight line approximation so we confined $r=\frac{b}{\sin \varphi}$ to $\left(0^{t h}\right)$ order.

$$
\dot{\varphi}=-\lim _{\dot{R} \rightarrow 0} \int_{0}^{\pi} \int_{\frac{b}{\sin \vartheta}}^{\dot{R}} \mathcal{K} d S
$$

Taking into count of Eq. (16), value of the photon's deflection angle in framework of plasma is calculated as;

$$
\dot{\varphi} \approx \frac{4 M}{b}-\frac{16 M \tilde{\omega}}{3 b^{3}}+\frac{2 M \omega_{e}^{2}}{b \omega_{\infty}^{2}}-\frac{4 M \tilde{\omega} \omega_{e}^{2}}{b^{3} \omega_{\infty}^{2}}+\mathcal{O}\left(M^{2}, \tilde{\omega}^{2}, \psi\right)
$$




\section{GRAPHICAL REPRESENTATION FOR PLASMA MEDIUM}

To this part, now we are going to check the deflection angle influences on graphically in context of plasma medium. Also inspect the physical impression of these graphs to visualize the behavior of the plasma surroundings. So, choose $M=1$ and the value of $\frac{\omega_{e}}{\omega_{\infty}}=10^{-1}$ and alternate value of $b$ (impact parameter), also fixed parameter to achieving such graphics.

A. Correspondence of the deflection angle $\dot{\varphi}$ with (impact parameter) $b$

(i)

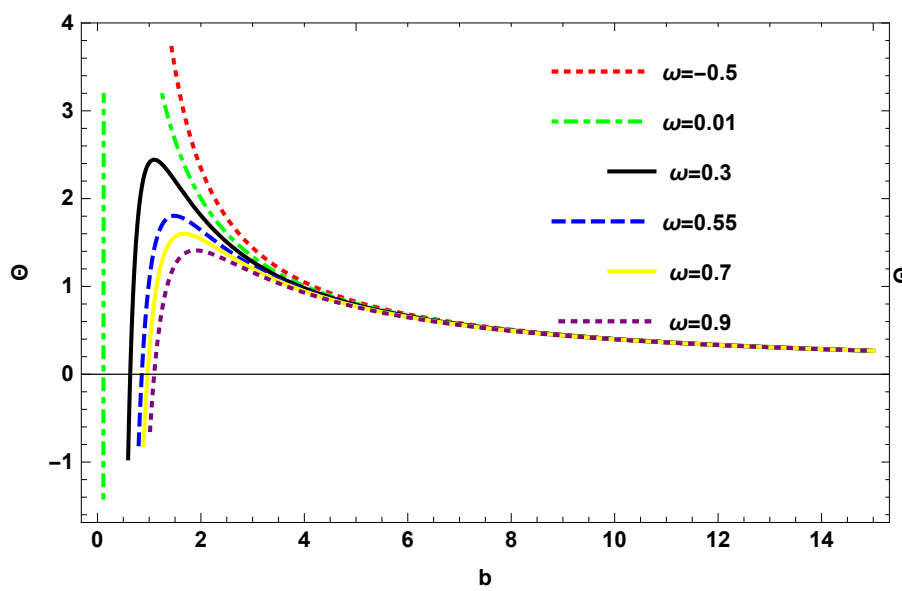

(ii)

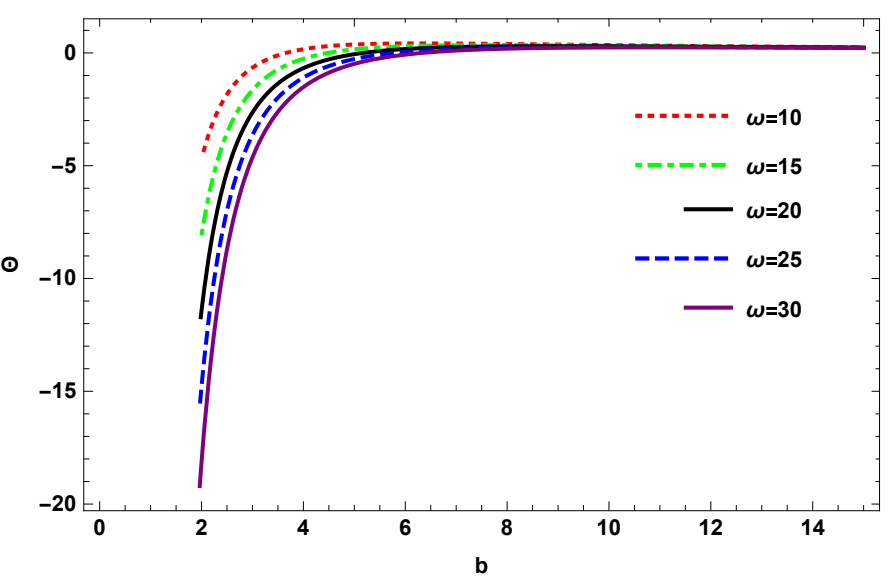

Picture 5: Relationship between $\varphi$ and (impact parameter).

- Picture 4 To demonstrate influence of the $\varphi$ with regards to parameter $b$ being fixed $M$ and varying $\omega$.

1. Picture (i), we viewed the deflection angle steadily increasing for letting smaller values $\omega$ and move towards +ve side.

2. Picture (ii), we determined deflection angle decreasing gradually for large values of $\omega$.

\section{B. Deflection angle $\varphi$ with resect to parameter $\omega$}

(i)

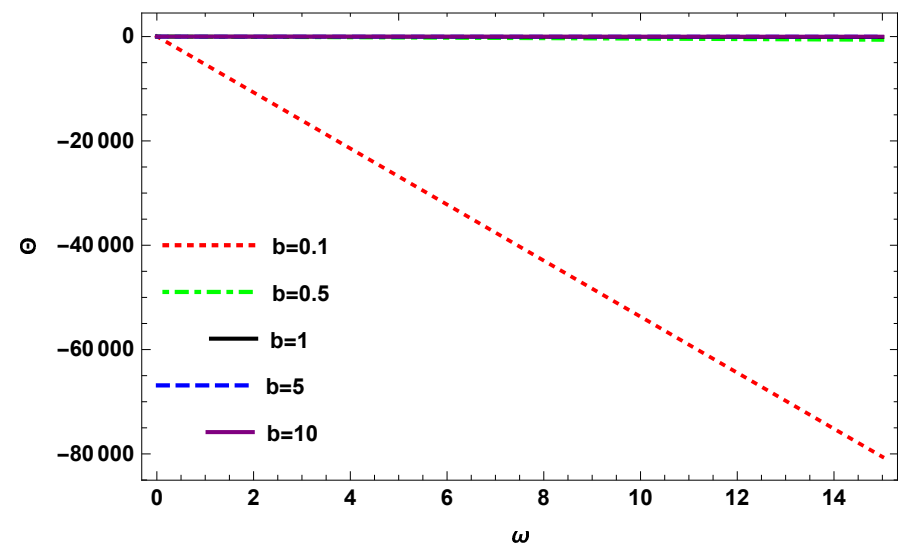

Picture 6: Relationship between $\varphi$ and $\omega$.

- Picture 6 Presents influence of the deflection angle with comparison to parameter $\omega$ for being fixed value of $M$ and changing the input values of impact parameter.

1. Picture (i); we analyzed that the value of deflection angle firstly decrease keeping the small to large values of $b$ and then moves toward positive side. 


\section{GREYBODY FACTOR FOR IMPROVED SCHWARZSCHILD BLACK HOLE}

The Schwarzschild metric in 4-dimension is given as [85];

$$
d s^{2}=-f(r) d t^{2}+\frac{d r^{2}}{f(r)}+r^{2}\left(d \theta^{2}+\sin ^{2} \theta d \phi^{2}\right)
$$

where,

$$
f(r)=1-\frac{\tilde{\omega}^{2}}{r^{4}}-\frac{2 \tilde{\omega}^{2} \psi M}{r^{5}}-\frac{2 M}{r}+\frac{2 M \tilde{\omega}}{r^{3}}
$$

For event horizon, we take $\psi=0$ and obtain the following;

$$
r_{h}=M \pm \sqrt{M^{2}-\tilde{\omega}}, \quad \pm \sqrt{\tilde{\omega}}
$$

The Schrodinger-like equation is illustrated as;

$$
\left(\frac{d^{2}}{d r_{*}^{2}}+\omega^{2}-\check{V}(r)\right) \phi(r)=0
$$

where, $r_{*}^{2}$ indicate the "tortoise coordinate".

$$
d r_{*}^{2}=\frac{1}{f(r)} d r
$$

and, potential $\check{V}(r)$ is given by

$$
\check{V}(r)=\frac{f(r) f^{\prime}(r)}{r}+l(l+1) \frac{f(r)}{r^{2}}
$$

To compute the lower bound base upon transmission probability with $h=\omega$ is [77];

$$
\begin{aligned}
T & \geq \frac{1}{\cosh ^{2}}\left(\frac{1}{2 \omega} \int_{-\infty}^{\infty} \check{V}(r) d r_{*}^{2}\right) \\
& =\frac{1}{\cosh ^{2}}\left[\frac{1}{2 \omega} \int_{r_{h}}^{\infty}\left(\frac{f^{\prime}(r)}{r}+\frac{l(l+1)}{r^{2}}\right) d r\right] \\
& =\frac{1}{\cosh ^{2}}\left[\frac{1}{2 \omega}\left(\frac{M}{r_{h}^{2}}-\frac{3 M \tilde{\omega}^{2}}{2 r_{h}^{4}}+\frac{4 \tilde{\omega}^{2}}{5 r_{h}^{5}}+\frac{l(l+1)}{r_{h}}\right)\right]
\end{aligned}
$$

substituting the $r_{h}$ value in above expression, we get the bound as;

$$
\begin{aligned}
T & \geq \frac{1}{\cosh ^{2}}\left[\frac { 1 } { 2 \omega } \left(\frac{M}{\left(M+\sqrt{M^{2}-\tilde{\omega}}\right)^{2}}-\frac{3 M \tilde{\omega}}{2\left(M+\sqrt{M^{2}-\tilde{\omega}}\right)^{4}}+\frac{4 \tilde{\omega}^{2}}{5\left(M+\sqrt{M^{2}-\tilde{\omega}}\right)^{4}}\right.\right. \\
& \left.\left.+\frac{l(l+1)}{\left(M+\sqrt{M^{2}-\tilde{\omega}}\right)}\right)\right]
\end{aligned}
$$

Hence, we have computed the lower bound for 4-dimensional improved Schwarzschild BH. If the BHs contain no electric charge or magnetic charge and take $\tilde{\omega}=0$, then given above bound is reduced into the form;

$$
T \geq \frac{1}{\cosh ^{2}}\left[\frac{2 \ell(\ell+1)}{8 \omega G M}\right]
$$

which shows the exactly same bound value for $4 D$ Schwarzschild (BH), emitting the spinless particles [77]. 


\section{GRAPHICAL INFLUENCES OF GREYBODY FACTOR}

In this section, we inspect the graphical importance of greybody factor of lower bound for improved Schwarzschild $\mathrm{BH}$ along with potential by assuming $(G=M=1)$ and alternating values of parameter $\tilde{\omega}$ while angular momentum $(\ell=0,1,2)$ respectively. For graph purpose we set $\tilde{\omega}=\alpha$.

(i)

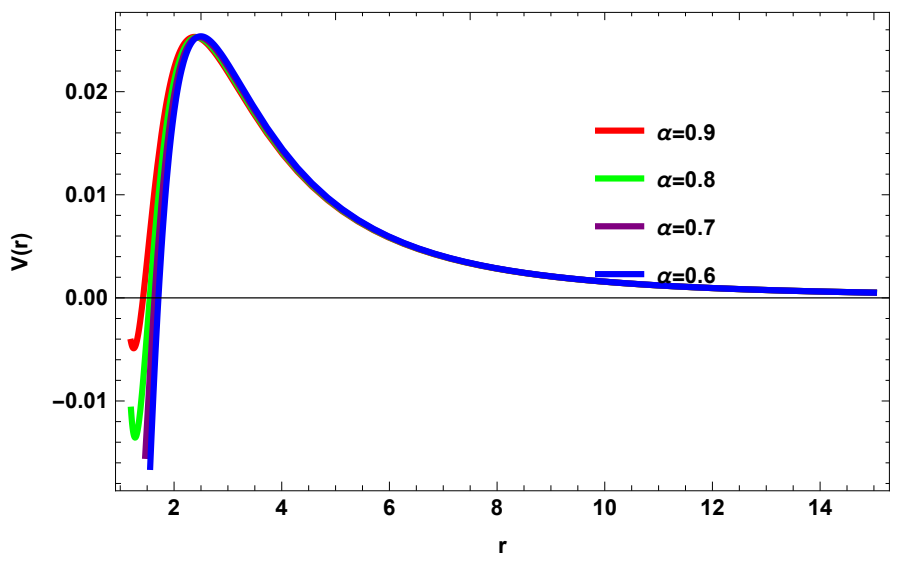

(i)

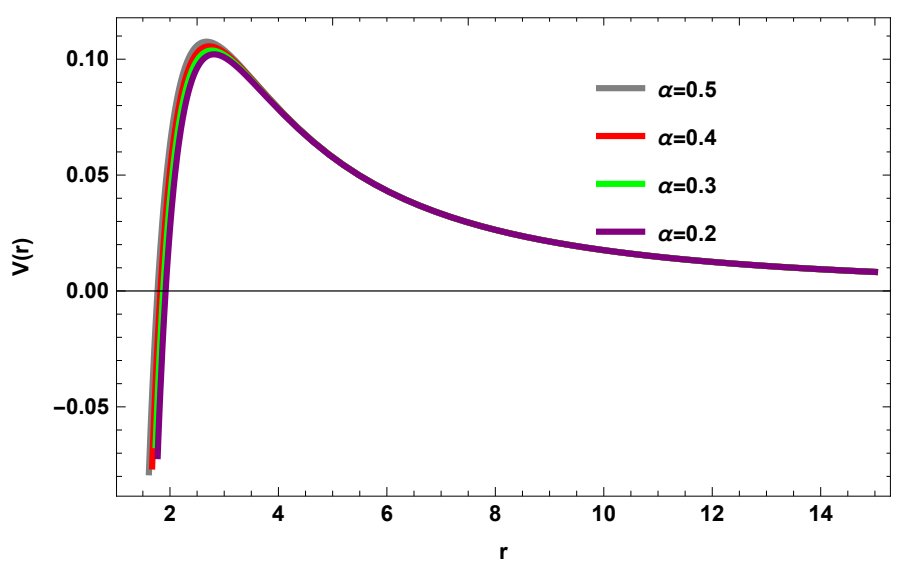

(i)

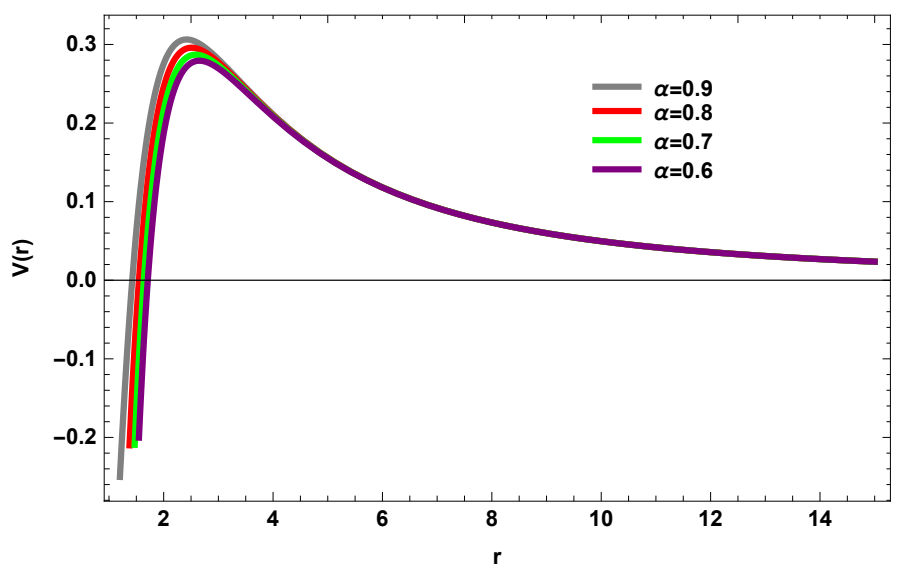

(ii)

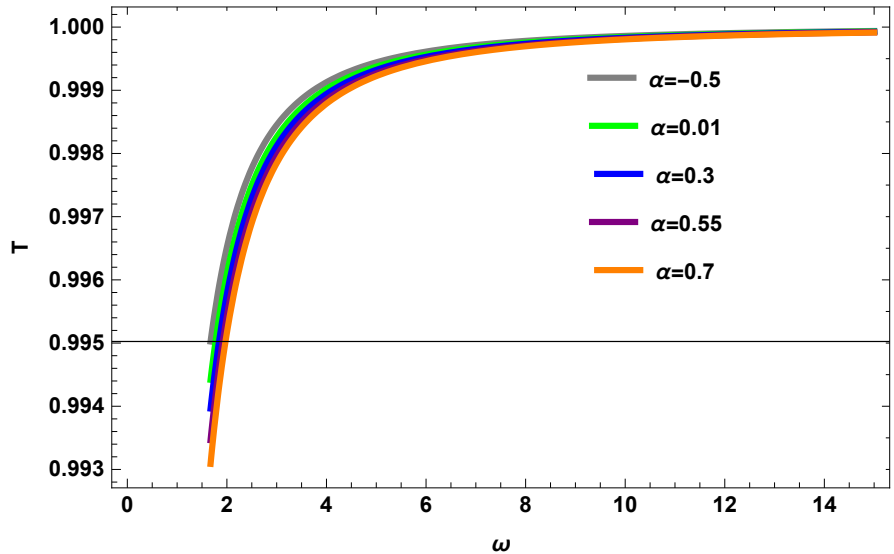

$\underset{\text { (ii) }}{\omega}$
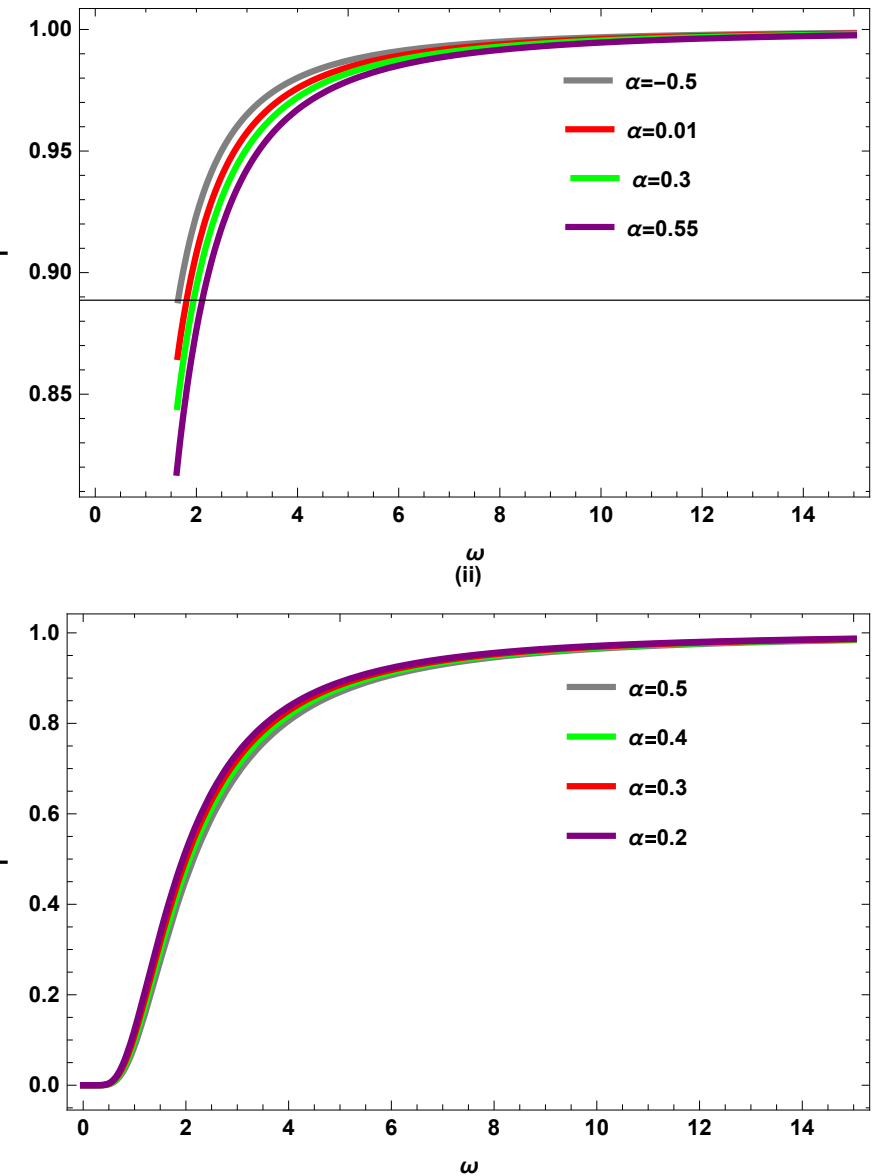

Figure 7: The left plot demonstrates the potential for improved Schwarzschild (BH) and the right plot shows the respective greybody factor of lower bound.

Here, we can observe how the influence of $T$ relates to the potential shape. This may achieve by alternating the free parameter $\psi$ values and the angular momentum $\ell$ parameter as well. By assuming $M$ and $G$ equal to 1 , the potential reaches at the higher peaks when $\psi$ changes which is shown in the left plot of Fig.7. The greybody factor gets the 
lower position for the provided value of $\omega$ although the wave face difficulty to transmit across the higher potential which shown in the right plot of Fig.7.

\section{SUMMARY}

In the recent doings, we briefly inspected the deflection angle for the improved Schwarzschild BH . In order to do so, we consider about the most valuable tools Gauss-Bonnet theorem designed by GW, we merely investigated the term deflection angle for the improved Schwarzschild BH. Taking the help of GBT, we find out the result for deflection angle of photon's with the comprising of a domain region outward of $b$ (impact parameter), which represents the gravitational lensing having a worldwide effect and has a strong equipment which probe on the prime of the singularities of $\mathrm{BH}$. As a result of our examination, we determine the value of deflection angle of the photons for thee improved Schwarzschild $\mathrm{BH}$ in weak fields limits by applying the procedure of Gauss-Bonnet theorem. As a result, the required value of deflection angle is described as

$$
\grave{\varphi} \approx \frac{4 M}{b}-\frac{16 M \tilde{\omega}}{3 b^{3}}+\mathcal{O}\left(M^{2}, \tilde{\omega}^{2}, \psi, r^{6}\right)
$$

By letting $\tilde{\omega}=0$ given of the above equation then our calculated value of deflection angle turned in the basic Schwarzschild deflection angle up to first order. Also examined the graphically significance of the deflection angle to improved Schwarzschild BH. Similarly, we derived deflection angle of photon's ray for improved Schwarzschild $\mathrm{BH}$ in the availability of plasma medium. The expression for the value of deflection angle of photon's ray to the influence of plasma is written as;

$$
\dot{\varphi} \approx \frac{4 M}{b}-\frac{16 M \tilde{\omega}}{3 b^{3}}+\frac{2 M \omega_{e}^{2}}{b \omega_{\infty}^{2}}-\frac{4 M \tilde{\omega} \omega_{e}^{2}}{b^{3} \omega_{\infty}^{2}}+\mathcal{O}\left(M^{2}, \tilde{\omega}^{2}, \psi\right)
$$

In order to remove the factor of plasma impact $\left(\frac{\omega_{e}}{\omega_{\infty}} \rightarrow 0\right)$, this equation is changed into above equation.

Further, we discuss about different graphs of the values of deflection angle to the background of plasma and then compare these graphs with vacuum's graphs. Also, we examined the greybody factor of improved Schwarzschild $(\mathrm{BH})$ by utilizing the rigorous bound. In this way, we properly study the improved Schwarzschild BH. Initially, we derive the horizon value and afterwards the Schrödinger-like equation is calculated for the radial part of the solution. Consequently, this permit us to examine the behavior of potential for determining the greybody factor. We obtained that the potential height down when lower the parameter $\alpha$ values. Moreover, the rigorous bound which is based upon the greybody factor have computed. So, we examine qualitatively, greybody factor lower bound by taking into account of the following potential frame; At the higher value of potential, the wave has faced the difficulty in order to transmit which produce the lower greybody factor bound.

[1] G.E. Romero and G.S. Vila, (Springer, 2014).

[2] J. Soldner, Ueber die Ablenkung eines Lichtstrals von seiner ger adlinigen Bewegung, durch die Attraktion eines Weltkörpers, an welchem er nahe vorbei geht. Berliner Astronomisches Jahrbuch, 161-172.cc(1804).

[3] S. Schaffer, Journal for the History of Astronomy 10, 42 (1979).

[4] M. Bartelmann and P. Schneider, Phys. Rep. 340, 291 (2001).

[5] M. Bartelmann, Gravitational lensing. Class. Quant. Gravit. 27, 233001 (2010).

[6] C.R. Keeton, C.S. Kochanek, and E.E. Falco, Astrophys. J. 509, 561 (1998).

[7] E.F. Eiroa, G.E. Romero, and D.F. Torres, Phys. Rev. D 66, 024010 (2002).

[8] S. Mao, B. Paczynski, Astrophys. J. 374, L37 (1991).

[9] V. Bozza, Phys. Rev. D 66, 103001 (2002).

[10] M. Sharif and S. Iftikhar, Astrophys. Space Sci. 357(1), 85 (2015).

[11] G.W. Gibbons and M.C. Werner, Class. Quant. Grav. 25, 235009 (2008).

[12] M.C. Werner, Gen. Relat. Gravit. 44, 3047 (2012).

[13] A. Ishihara, Y. Suzuki, T.Ono, T. Kitamura and H.Asada, Phys. Rev. D 94(8), 084015 (2016).

[14] G. Crisnejo and E. Gallo, Phys. Rev. D 97, no.12, 124016 (2018).

[15] K. Jusufi, M.C. Werner, A. Banerjee and A. Övgün, Phys. Rev. D 95(10),104012 (2017).

[16] K. de Leon and I. Vega, Phys. Rev. D 99, no.12, 124007 (2019).

[17] I. Sakalli and A. Övgün, EPL 118(6), 60006 (2017).

[18] K. Jusufi and A. Övgün, Phys. Rev. D 97(2), 024042 (2018).

[19] T. Ono, A. Ishihara and H. Asada, Phys. Rev. D 96(10), 104037 (2017). 
[20] K. Jusufi, A. Övgün and A. Banerjee, Phys. Rev. D 96, no.8, 084036 (2017).

[21] A. Övgün, G. Gyulchev and K. Jusufi, Ann. Phys. 406, 152-172 (2019).

[22] K. Jusufi, I. Sakalli, and A.Ovgun, Phys. Rev.D 96 (2), 024040 (2017).

[23] H. Arakida, Gen. Relativ. Gravit.50(5), 48 (2018).

[24] T. Ono, A. Ishihara, and H. Asada, Phys. Rev. D 98(4), 044047 (2018).

[25] K. Jusufi and A. Övgün, Phys. Rev. D 97(6),064030 (2018).

[26] A. Övgün, K. Jusufi and I. Sakalli, Phys. Rev. D 99(2), 024042 (2019).

[27] K. Jusufi, A. Övgün, J. Saavedra, Y. Vasquez and P. A. Gonzalez, Phys. Rev. D 97, no.12, 124024 (2018).

[28] A. Övgün, Phys. Rev. D 98(4), 044033 (2018).

[29] A. Övgün, K. Jusufi and I. Sakalli, Ann. Phys. 399, 193 (2018).

[30] A. Övgün, Universe 5(5), 115 (2019).

[31] A. Övgün, İ. Sakallı and J. Saavedra, Annals Phys. 411, 167978 (2019).

[32] A. Övgün, I. Sakalli and J. Saavedra, JCAP 1810(10), 041 (2018).

[33] T. Ono, A. Ishihara and H. Asada, Phys. Rev. D 99(12), 124030 (2019).

[34] A. Övgün, Phys. Rev. D 99(10),104075 (2019).

[35] W. Javed, R. Babar and A. Övgün, Phys. Rev. D 99(8), 084012 (2019).

[36] A. Övgün and İ. Sakallı, Annals Phys. 413, 168071 (2020).

[37] A. Övgün, Phys. Lett. B 820, 136517 (2021).

[38] K. Jafarzade, M. Kord Zangeneh and F. S. N. Lobo, JCAP 04, 008 (2021).

[39] K. Takizawa, T. Ono and H. Asada, Phys. Rev. D 101, no.10, 104032 (2020).

[40] Y. Kumaran and A. Övgün, Chin. Phys. C 44, no.2, 025101 (2020).

[41] Z. Li and A. Övgün, Phys. Rev. D 101, no.2, 024040 (2020).

[42] Z. Li, G. Zhang and A. Övgün, Phys. Rev. D 101, no.12, 124058 (2020).

[43] K. Jusufi, A. Övgün, A. Banerjee and ·. I. Sakallı, Eur. Phys. J. Plus 134, no.9, 428 (2019).

[44] A. Övgün, Turk. J. Phys. 44, no.5, 465-471 (2020)

[45] Y. Kumaran and A. Övgün, Turk. J. Phys. 45, 247-267 (2021)

[46] M. Okyay and A. Övgün, [arXiv:2108.07766 [gr-qc]]

[47] R. C. Pantig, P. K. Yu, E. T. Rodulfo and A. Övgün, [arXiv:2104.04304 [gr-qc]].

[48] K. Takizawa, T. Ono and H. Asada, Phys. Rev. D 102, no.6, 064060 (2020).

[49] T. Ono and H. Asada, Universe 5, no.11, 218 (2019).

[50] Q. M. Fu, L. Zhao and Y. X. Liu, Phys. Rev. D 104, no.2, 024033 (2021).

[51] A. Ishihara, Y. Suzuki, T. Ono and H. Asada, Phys. Rev. D 95, no.4, 044017 (2017).

[52] H. El Moumni, K. Masmar and A. Övgün, [arXiv:2008.06711 [gr-qc]].

[53] W. Javed, R. Babar, and A. Övgün, Phys. Rev. D 100, no.10, 104032 (2019).

[54] W. Javed, J. Abbas, and A. Övgün, Eur. Phys. J. C 79, no.8, 694 (2019).

[55] W. Javed, J. Abbas and A. Övgün, Phys. Rev. D 100, no.4, 044052 (2019).

[56] W. Javed, A. Hazma and A. Övgün,Phys. Rev. D 101, no.10, 103521 (2020).

[57] W. Javed, M. B. Khadim, A. Övgün and J. Abbas, Eur. Phys. J. Plus 135, no.3, 314 (2020).

[58] W. Javed, M. B. Khadim and A. Övgün, Eur. Phys. J. Plus 135, 595 (2020).

[59] W. Javed, A. Hamza and A. Övgün, Mod. Phys. Lett. A 35, 2050322 (2020).

[60] W. Javed, A. Hamza and A. Övgün, Universe $7,10,385$ (2021).

[61] W. Javed, J. Abbas, Y. Kumaran and A. Övgün, Int. J. Geom. Meth. Mod. Phys. 18, 2150003 (2021).

[62] W. Javed, J. Abbas and A. Övgün, Ann. Phys. 418, 168183 (2020).

[63] S. W. Hawking, Commun. Math. Phys. 43 (1975), 199-220[erratum: Commun. Math. Phys. 46 (1976), 206].

[64] E. T. Akhmedov, V. Akhmedova and D. Singleton, Phys. Lett. B 642, 124-128 (2006).

[65] W. Javed, R. Ali, R. Babar and A. Övgün, Chin. Phys. C 44, no.1, 015104 (2020).

[66] I. Sakalli and A. Övgün, Gen. Rel. Grav. 48, no.1, 1 (2016).

[67] X. M. Kuang, B. Liu and A. Övgün, Eur. Phys. J. C 78, no.10, 840 (2018).

[68] I. Sakalli and A. Ovgun, Eur. Phys. J. Plus 130, no.6, 110 (2015).

[69] W. Javed, G. Abbas and R. Ali, Eur. Phys. J. C 77, no.5, 296 (2017).

[70] S. W. Hawking, Phys. Rev. D 13 191-197 (1976).

[71] S. Fernando, Gen. Relativ. Gravit. 37, 461(2005).

[72] J. Escobedo, M.S. thesis, University of Amsterdam(2008).

[73] C. H. Fleming, (2005),http:/ / www.physics.umd.edu/grt/taj/776b/fleming.pdf.

[74] P. Lange, M.S. thesis, Uppsala Universitet(2007).

[75] P. Boonserm and M. Visser, “Bounding the Bogoliubov coefficients," Annals Phys. 323, 2779-2798 (2008).

[76] P. Boonserm, "Rigorous bounds on Transmission, Reflection, and Bogoliubov coefficients," [arXiv:0907.0045 [math-ph]].

[77] P. Boonserm and M. Visser, Phys. Rev. D 78, 101502 (2008).

[78] T. Ngampitipan and P. Boonserm, Int. J. Mod. Phys. D 22, 1350058 (2013).

[79] P. Boonserm, T. Ngampitipan and P. Wongjun, Eur. Phys. J. C 78, no.6, 492 (2018).

[80] P. Boonserm, T. Ngampitipan and M. Visser, JHEP 03, 113 (2014).

[81] P. Boonserm, A. Chatrabhuti, T. Ngampitipan and M. Visser, J. Math. Phys. 55, 112502 (2014).

[82] T. Ngampitipan and P. Boonserm, J. Phys. Conf. Ser. 435, 012027 (2013).

[83] P. Boonserm, T. Ngampitipan and P. Wongjun, Eur. Phys. J. C 79, no.4, 330 (2019). 
[84] S. Kanzi, S. H. Mazharimousavi and İ. Sakallı, Annals Phys. 422, 168301 (2020).

[85] A. Bonanno and M. Reuter, Phys. Rev. D 62, 043008 (2000).

[86] Á. Rincón and G. Panotopoulos, Phys. Dark Univ. 30, 100639 (2020) 